LBL- 31802

DE92 008316

\title{
AN EXPERIMENTAL REVIEW OF HYPERON MAGNETIC MOMENTS †
}

\author{
K. B. Luk \\ Department of Physics \\ University of Callifornia \\ and \\ Physics Division \\ Lawrence Berkeley Laboratory \\ University of Callifornia \\ Berkeley, California 94720, U.S.A.
}

January, 1992

+ Invited talk presented at the 20th International Symposium on Hypernuclear and Strange Particle Physics, Shimoda, Japan, Dec 9-12, 1991.

This work was supported by the Director, Office of Energy Research, Office of High Energy and Nuclear Physics, Division of High Energy Physics, of the U.S. Department of Energy under Contract No. DEAC03-76SF0098. 


\title{
AN EXPERIMENTAL REVIEW OF HYPERON MAGNETIC MOMENTS
}

\author{
K. B. Luk
}

Department of Physics and Lawrence Berkeley Laboratory, University of California, Berkeley, California 94720, U.S.A.

\begin{abstract}
Hyperon magnetic moments are important probes for studying the structure of baryons. In this talk, I shall briefly describe how the measurements are made and discuss the current status of the determinations.
\end{abstract}

\section{INTRODUCTION}

One of the fundamental entities of a particle is its magnetic moment, $\mu$, which is defined as

$$
\mu=g \frac{q}{2 M} S
$$

where $M$ is the mass, $S$ is the spin, $q$ is the electric charge, and $g$ is the gyromagnetic factor of the particle. For a point-like charged particle, $g$ is 2 . If the particle is electrically meutral, then $g$ is expected to be zero. Any deviation from these g-values is called the anomalous magnetic moment of the particle, $k$.

The importance of knowing the magnetic moment of a particle is easily realized when one compares the extremely precise measured g-factors of tha electron and the muon ${ }^{1}$ to the QED calculations: ${ }^{2}$

$$
\begin{aligned}
& \mathrm{g}_{e_{\mathrm{Exp}}}^{\text {Exp }}=2.002319304386 \pm 0.000000000020 \\
& \mathrm{~g}_{e}^{\mathrm{QED}}=2.0023193048 \pm 0.0000000008
\end{aligned}
$$




$$
\begin{aligned}
& \mathrm{g}_{\mu}^{\text {Expt }}=2.002331846 \pm 0.000000016 \\
& \mathrm{~g}_{\mu}^{\text {QED }}=2.002331836 \pm 0.000000020
\end{aligned}
$$

The agreement between the measurements and the predictions is excellent. These measurements yield one of the most stringent tests of QED. Furthermore, they can also be used to probe physics beyond the Standard Model.

In the hadron sector, the existence of non-zero anomalous magnetic moments for the proton and neutron, $\kappa_{p}=1.793$ and $\kappa_{n}=-1.913$ respectively, provided the first evidence that hadrons are composite, making up of quarks which are described by QCD. Thus precise determination of the hyperon magnet moments holds the promise of offering us insights into the hadronic structure:

\section{EXPERIMENTAL METHODS AND RESULTS}

The magnetic moments of the ground state hyperons so far have been determined by three methods. The transition moment of $\Sigma^{0} \rightarrow \Lambda, \mu_{\Sigma \Lambda}$, is measured by the Primakoff process. Sometimes a magnetic monnent can be extracted by studying the transitions between energy levels in exotic atoms. Nevertheless, the most commonly used technique is the classic spin precession method.

\subsection{Primakoff effect}

The Primakoff effect allows the determination of $\mu_{\Sigma \Lambda}$ from the cross section for $\Lambda \rightarrow \Sigma^{0}$ in the nuclear Coulomb field. Figure 1 is an illustration of the reaction, where $Z$ is the atomic number of the target material.

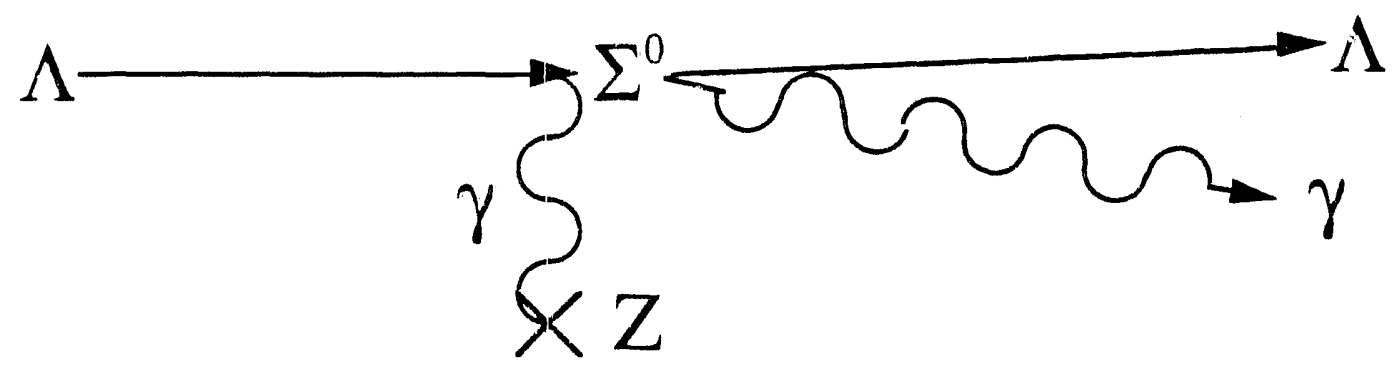

Figure 1. Primakoff Production of $\Sigma^{0}$ from $\Lambda$ 
The $\Sigma^{0}$ is primarily produced with very little transverse momentum. So far there are two determinations of the transition moment, yielding $\left|\mu_{\Lambda \Sigma}\right|=1.72_{-0.19}^{+0.17}$ nuclear magnetons (n.m.) by Dydak et al. at CERN, ${ }^{3}$ and $1.59 \pm 0.05 \pm 0.07$ n.m. by Petersen et al. at Fermilab. ${ }^{4}$ Since the total cross section of the process is proportional to $\left(\mu_{\Sigma \Lambda}\right)^{2}$, the sign of the measu $d \mu_{\Sigma \Lambda}$ must be taken from the quark model prediction. The weighted average of the two measurements is $-1.61 \pm 0.08$ n.m.

\subsection{Exotic atom method}

An exotic atom is formed when a negatively charged baryon is captured by an atom. For such an atom, the fine-structure splitting of the atomic level is given by

$$
\delta E_{n, 1}=(-1+\kappa)(Z \alpha)^{4} \frac{M}{2 n^{3} 1(1+1)}
$$

where $\mathrm{n}$ and $\mathrm{l}$ are the principle and orbital angular momentum quantum number respectively, $M$ is the reduced mass of the atom, and $\alpha$ is the fine structure constant. By examining the $\mathrm{X}$-rays emitted in the transitions between various atomic levels, given by equation (2), the magnetic moment of the captured baryon can then be determined. This method has been applied to $\Sigma^{-}$(as well as $\bar{p}$ ). The problem with this technique is that it requires a fair amount of atomic physics corrections. Also, the experiments did not have sufficient energy resolution to resolve the sign of the $\Sigma^{-}$magnetic moment. The best determination of the $\Sigma^{-}$magnetic moment performed with the $\Sigma^{-}$-exotic atom came from Hertzog et al. at $B N L,{ }^{5} \mu_{\Sigma^{-}}=-1.105 \pm 0.029 \pm 0.010$ n.m., where the sign is taken from other measurernents using the spin precession method.

\subsection{Spin precession approach}

In this method the hyperon magnetic moment is determined by measuring the spin precession angle of a polarized sample of hyperons in a uniform magnetic field. For the neutral hyperon the precession angle, $\phi$, is given by ${ }^{6}$

$$
\phi=\frac{2 \mu}{\beta} \int \mathrm{B} d l
$$


whereas for a charged particle,

$$
\phi=\frac{q}{M \beta}\left(\frac{g}{2}-1\right) \int B d l
$$

with $\beta$ being the speed of the hyperon in unit of $c$, and $\int B d l$ the field integral. In practice, the final orientation of the polarization is determined by studying the angular distribution of the hyperon decay. Once the initial direction of the polarization is know; $\phi$ can be calculated.

There are three important factors that make the spir. precession method particularly suitable for carrying out precise hyperon magnetic moment measurements at high energy. A few hundred $\mathrm{GeV}$ hyperon beam typically has a mean decay length of the order of ten meters in the laboratory frame, sufficient to traverse a significant portion of strong magnetic field now available. At high energy relatively intense hyperon beams are easily prepared. Furthermore, all hyperons including $\bar{\Xi}^{+}$, but not $\Omega^{-}$or $\bar{\Lambda}$, produced by high energy unpolarized protons incident on an unpolarized target at finite angle are found to possess significant polarization, $10-25 \%$ at $\mathrm{p}_{\mathrm{t}}$ of about $1 \mathrm{GeV} / \mathrm{c}$. Figures 2 and 3 show the polarization of $\Lambda, \Xi, \Sigma$ and $\Xi^{+}$.

There has been no new determination of the neutral hyperon magnetic moment since the late seventies. Our current knowledge of the $\Lambda$ and $\Xi^{0}$ magnetic moments primarily comes from one to two precise measurements. ${ }^{7,8}$

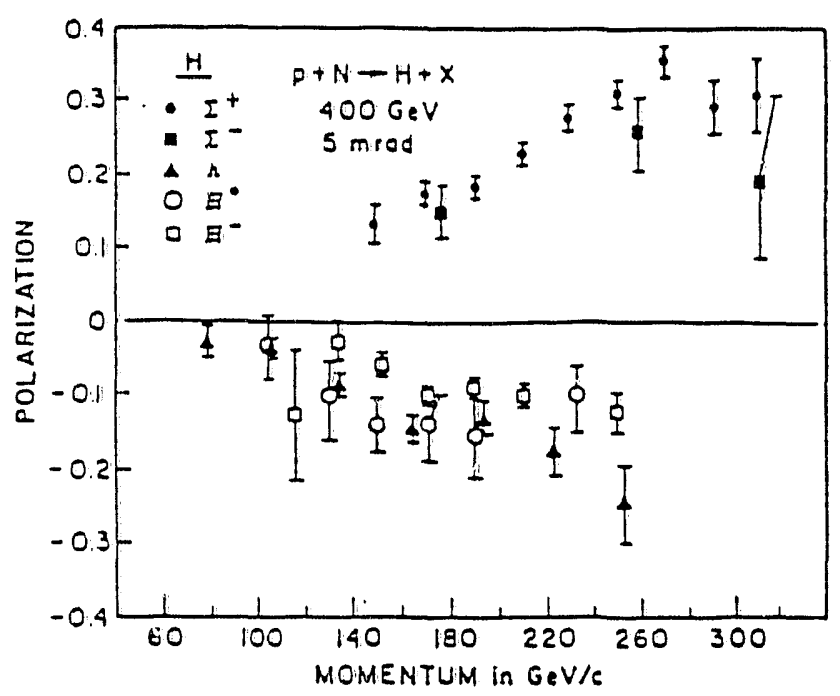

Figure 2. Production polarization of $\Lambda, \Xi$ and $\Sigma$ hyperons in $400 \mathrm{GeV}$ p-N reactions at 5 mrad as a function of hyperon momentum. 


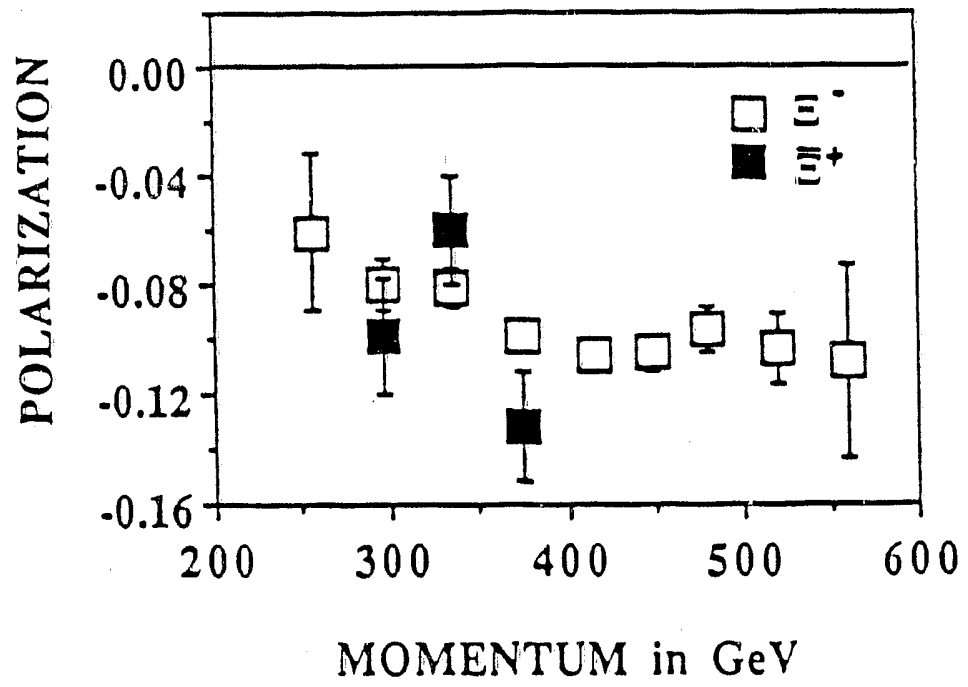

Figure 3. Polarization of $\Xi^{+}$and $\Xi$ - hyperons produced by $800 \mathrm{GeV}$ protons at $2.5 \mathrm{mrad}$ as a function of hyperon momentum.

For $\Sigma^{+}$, the situation is confusing. The Fermilab experiments E497 and E620 determined $\mu_{\Sigma^{+}}$to be $2.38 \pm 0.02$ n.m. and $2.479 \pm 0.012 \pm 0.022$ n.m. respectively, ${ }^{9}$ both with a precision of about $1 \%$ but disagree from each other by 3.1 standard deviations. It is desirable to resolve the discrepancy.

In the case of $\Sigma^{-}$, Fermilab E620, E497 and E715 used the decay $\Sigma \rightarrow n \pi^{-}$to determine the final direction of the $\Sigma^{-}$polarization. Due to the small value of the decay parameter, $\alpha_{n \pi}$, measuring the polarization components for finding the precession angle without any systematics is non-trivial. E715 also made use of the $\Sigma^{-} \rightarrow n e^{-} \bar{v}$ decay, which has a larger decay parameter, to cross check the measurement. Their final result for the $\Sigma$ magnetic moment is $-1.166 \pm 0.014 \pm 0.010 \mathrm{n} . \mathrm{m}$., which should be compared with $-0.89 \pm 0.14$ n.m. and $-1.23 \pm 0.03 \pm 0.03$ n.m. from E620 and E497 respectively. ${ }^{10}$ The weighted average of these three determinations is $-1.171 \pm 0.016$ n.m., approximately two standard deviations away from the one measured with exotic atoms. Again, it would be nice to have new measurements to pin down the $\Sigma^{-}$magnetic moment.

Recently, based on 70,000 events, Fermilab E756 provided the first determination of the $\Xi^{+}$magnetic moment which is found to be $0.657 \pm 0.028 \pm 0.020$ n.m.. ${ }^{11}$ This experiment also has the best $\Xi^{-}$magnetic moment measurement to date. With 4.36 millions $\Xi^{-1} \mathrm{~s}, \mu_{\Xi}$. is determined to be $-0.6505 \pm 0.0025$ n.m. ${ }^{12}$ consistent with the previous measurements. ${ }^{13}$ As expected, the $\Xi^{-}$and $\Xi^{+}$measurements are in good agreement with CPT invariance. 
In addition, E756 discovered that $\Omega^{-}$can not be polarized by protons in the 'standard' fashion. Subsequently they applied the spin transfer technique to generate polarized $\Omega^{-\prime}$ 's. A neutral beam rich in polarized $\Lambda^{\prime} s$ and $\Xi^{0}$ 's created from high energy unpolarized protons struck the $\Omega^{-}$production target at $0 \mathrm{mrad}$. $\Omega^{\prime \prime}$ s produced in this way were found to have sufficient polarization for making the first measurement of the $\Omega^{\circ}$ magnetic moment. The reported value for $\mu_{\Omega}$. is $-1.94 \pm 0.17 \pm 0.14$ n.m.. ${ }^{14}$

\section{FUTURE PROSPECTS}

In the next few years, new hyperon magnetic moment results are expected to come from two Fermilab experiments, E761 and E800, which have completed data taking.

With millions of $\Sigma^{+1}$ s, E761 will measure the $\Sigma^{+}$magnetic moment again. Hopefully, this will resolve the outstanding discrepancy of the existing results. In addition, E76.1 has tried out the idea of using crystal channelling to spin precess $\Sigma^{+}$s. This approach is applicable to particles with a much shorter lifetime than the ground state hyperons.

The preliminary result from E761 indicates that $\bar{\Sigma}$ is also polarized when produced by unpolarized protons. The magnitude of the polarization is about $10 \%$, similar to $\Xi^{+}$. This should provide the first measurement of the $\bar{\Sigma}$ magnetic moment.

E800 has collected sampies of polarized $\Omega^{-1}$ s produced by two different methods:

(1) applied the spin-transfer method as in E756, but with larger field integrals;

(2) made use of a high energy unpolarized neutral hyperon beam striking the $\Omega^{-}$ production target at an angle.

With a total number of approximately $250,000 \Omega$ 's, the anticipated statistical uncertainty in the $\Omega^{-}$magnetic moment is 0.03 n.m.. It should be precise enough to challenge any theoretical predictions.

\section{SUMMARY}

Table 1 is a summary of the current status of the baryon magnetic moment measurements. Except for $\mu_{\Sigma \Lambda}$ and $\mu_{\Omega}$., all hyperon magnetic moments have been determined to better than $2 \%$. Comparison with experimental results shows that quark models, QCD sum rule, and lattice gauge calculations can predict the sign of the hyperonmagnetic moments. However, the calculations have a difficult time in reproducing the magnitudes of the magnetic moments. In the worst case, the disagreement is about 0.25 n.m.. 
In the near future, we expect a better measurment of $\mu_{\Omega^{-}}$from E800. E761 should be able to resolve the discrepancy between the existing measurements of the $\Sigma^{+}$magnetic moment. They may provide the first measurement of the $\bar{\Sigma}$ magnetic moment, and may demonstrate spin precession by crystal channelling.

Table 1. Measurements of baryon magnetic moments. Some theoretical predictions are shown are for comparison.

\begin{tabular}{cccccc} 
& $\begin{array}{c}\text { Experiment } \\
\text { (n.m.) }\end{array}$ & $\begin{array}{c}\text { SU(6) } \\
\text { (n.m.) }\end{array}$ & $\begin{array}{c}\text { Cloudy } \\
\text { Bag }^{16}\end{array}$ & $\begin{array}{c}\text { QCD Sum } \\
\text { Rule }{ }^{17}\end{array}$ & $\begin{array}{l}\text { Lattice } \\
\text { Gauge }\end{array}$ \\
\hline $\mathrm{p}$ & $2.792847^{\dagger}$ & input & 2.60 & 3.0 & $2.3 \pm 0.3$ \\
$\mathrm{n}$ & $-1.913043^{\dagger}$ & input & -2.01 & -2.0 & $-1.3 \pm 0.2$ \\
$\Lambda$ & $-0.613 \pm 0.004^{\dagger}$ & input & -0.58 & -0.7 & $-0.40 \pm 0.07$ \\
$\Sigma^{+}$ & $2.42 \pm 0.05^{\dagger}$ & 2.67 & 2.34 & 2.4 & $1.9 \pm 0.2$ \\
$\Sigma^{-}$ & $-1.156 \pm 0.014^{\dagger}$ & -1.09 & -1.08 & -1.0 & $-0.87 \pm 0.09$ \\
$\Sigma \rightarrow \Lambda$ & $-1.61 \pm 0.08^{\dagger}$ & -1.63 & - & -1.56 & $-1.15 \pm 0.16$ \\
$\Xi^{0}$ & $-1.253 \pm 0.014^{\dagger}$ & -1.43 & -1.27 & -1.4 & $-0.95 \pm 0.08$ \\
$\Xi^{-}$ & $-0.6508 \pm 0.0025$ & -0.49 & -0.51 & -0.9 & $-0.41 \pm 0.06$ \\
$\Omega^{-}$ & $-1.94 \pm 0.22$ & -1.84 & - & - & $-1.7 \pm 0.5 *$
\end{tabular}

${ }^{+}$taken from reference 15

* taken from reference 19

\section{ACKNOWLEDGEMENT}

I would like to thank Professor K. Imai for inviting me to give this talk at the Symposium. I also wish to express my gratitude to the organizers, in particular Dr. O. Morimatsu and Professor K. Yazaki, for their warm hospitality. Fruitful discussions with my colleagues K. Heller, R. Rameika and J. Lach are greatly appreciated. This work was supported by the Director, Office of Energy Research, Office of High Energy and Nuclear Physics, Division of High Energy Physics, of the U.S. Department of Energy under Contract No. DE-AC03-76SF0098. 
In the near future, we expect a better measurment of $\mu_{\Omega^{-}}$from E800. E761 should be able to resolve the discrepancy between the existing measurements of the $\Sigma^{+}$magnetic moment. They may provide the first measurement of the $\bar{\Sigma}$ magnetic moment, and may demonstrate spin precession by crystal channelling.

Table 1. Measurements of baryon magnetic moments. Some theoretical predictions are shown are for comparison.

\begin{tabular}{|c|c|c|c|c|c|}
\hline & $\begin{array}{c}\text { Experiment } \\
\text { (n.m.) }\end{array}$ & $\begin{array}{l}\mathrm{SU}(6) \\
\text { (n.m.) }\end{array}$ & $\begin{array}{l}\text { Cloudy } \\
\text { Bag }\end{array}$ & $\begin{array}{l}\text { QCD Sum } \\
\text { Rule }^{17}\end{array}$ & $\begin{array}{l}\text { Lattice } \\
\text { Gauge }\end{array}$ \\
\hline $\mathrm{p}$ & $2.792847^{+}$ & input & 2.60 & 3.0 & $2.3 \pm 0.3$ \\
\hline$n$ & $-1.913043^{+}$ & input & -2.01 & -2.0 & $-1.3 \pm 0.2$ \\
\hline$\Lambda$ & $-0.613 \pm 0.004^{4}$ & input & -0.58 & -0.7 & $-0.40 \pm 0.07$ \\
\hline$\Sigma^{+}$ & $2.42 \pm 0.05^{\dagger}$ & 2.67 & 2.34 & 2.4 & $1.9 \pm 0.2$ \\
\hline$\Sigma$ & $-1.156 \pm 0.1014^{\dagger}$ & -1.09 & -1.08 & -1.0 & $-0.87 \pm 0.09$ \\
\hline$\Sigma \rightarrow \Lambda$ & $-1.61 \pm 0.018^{\dagger}$ & -1.63 & - & -1.56 & $-1.15 \pm 0.16$ \\
\hline$\Xi^{0}$ & $-1.253 \pm 0.014^{\dagger}$ & -1.43 & -1.27 & -1.4 & $-0.95 \pm 0.08$ \\
\hline$\Xi^{-}$ & $-0.6508 \pm 0.0025$ & -0.49 & -0.51 & -0.9 & $-0.41 \pm 0.06$ \\
\hline$\Omega^{-}$ & $-1.94 \pm 0.22$ & -1.84 & - & - & $-1.7 \pm 0.5^{*}$ \\
\hline
\end{tabular}

\section{ACKNOWLEDGEMENT}

I would like to thank Professor K. Imai for inviting me to give this talk at the Symposium. I also wish to express my gratitude to the organizers, in particular Dr. O. Morimatsu and Professor K. Yazaki, for their warm hospitality. Fruitful discussions with my colleagues K. Heller, R. Rameika and J. Lach are greatly appreciated. This work was supported by the Director, Office of High Energy and Nuclear Physics, Division of High Energy Physics, of the U.S. Department of Energy under Contract No. DE-AC0376 SF0098. 


\section{REFERENCES}

1. E. R. Cohen and B. N. Taylor, Rev. Mod. Phys. 59, 1121 (1987).

2. T. Kinoshita and W. B. Lindquist, Phys. Rev. D27, 853, 867, 877, 886 (1983);

T. Kinoshita, B. Nizic and Okamoto, Phys. Rev. Lett. 52, 717 (1984).

3. F. Dydak et al., Nucl. Phys. B118, 1 (1977).

4. P. C. Petersen et al., Phys. Rev. Lett. 57, 949 (1986).

5. D. W. Hertzog et al., Phys. Rev. D37, 1142 (1988).

6. V. Bargmann, L. Michel and V. A. Telegdi, Phys. Rev. Lett. 2, 435 (1959).

7. L. Schachinger et al., Phys. Rev. Lett. 41, 1348 (1978).

8. P. T. Cox et al., Phys. Rev. Lett. 46, 877 (1981).

9. C. Ankenbrandt et al., Phys. Rev. Lett. 51, 863 (1983);

C. Wilkinson et al., Phys. rev. Lett. 58, 855 (1987).

10. L. Deck et al., Phys. Rev. D28, 1 (1983);

Y. W. Wah et al., Phys. Rev. Lett. 55, 2551 (1985);

G. Zapalac et al., Phys. Rev. Lett. 57, 1526 (1986).

11. P. M. Ho et al., Phys. Rev. Lett. 65, 1713 (1990).

12. J. Duryea et al., to be published in Phys. Rev. Lett. (1992).

13. R. Rameika et al., Phys. Rev. Lett. 52, 581 (1984);

L. H. Trost et al., Phys. Rev. D40, 1703 (1.989).

14. H. T. Diehl et al., Phys. Rev. Lett. 67, 804 (1991).

15. J. J. Hernandez et al. (Particle Data Group), Phys. Lett. B239, 1 (1990).

16. S. Theberge and A. W. Thomas, Phys. Rev. D25, 284 (1982).

17. B. L. Ioffe and A. V. Smilga, Phys. Lett. 133B, 436 (1983).

18. D. B. Leinweber, R. M. Woloshyn and T. Draper, Phys. Rev. D43, 1659 (1991).

19. C. Bernard et al., Phys. Rev. Lett. 49, 1076 (1982). 


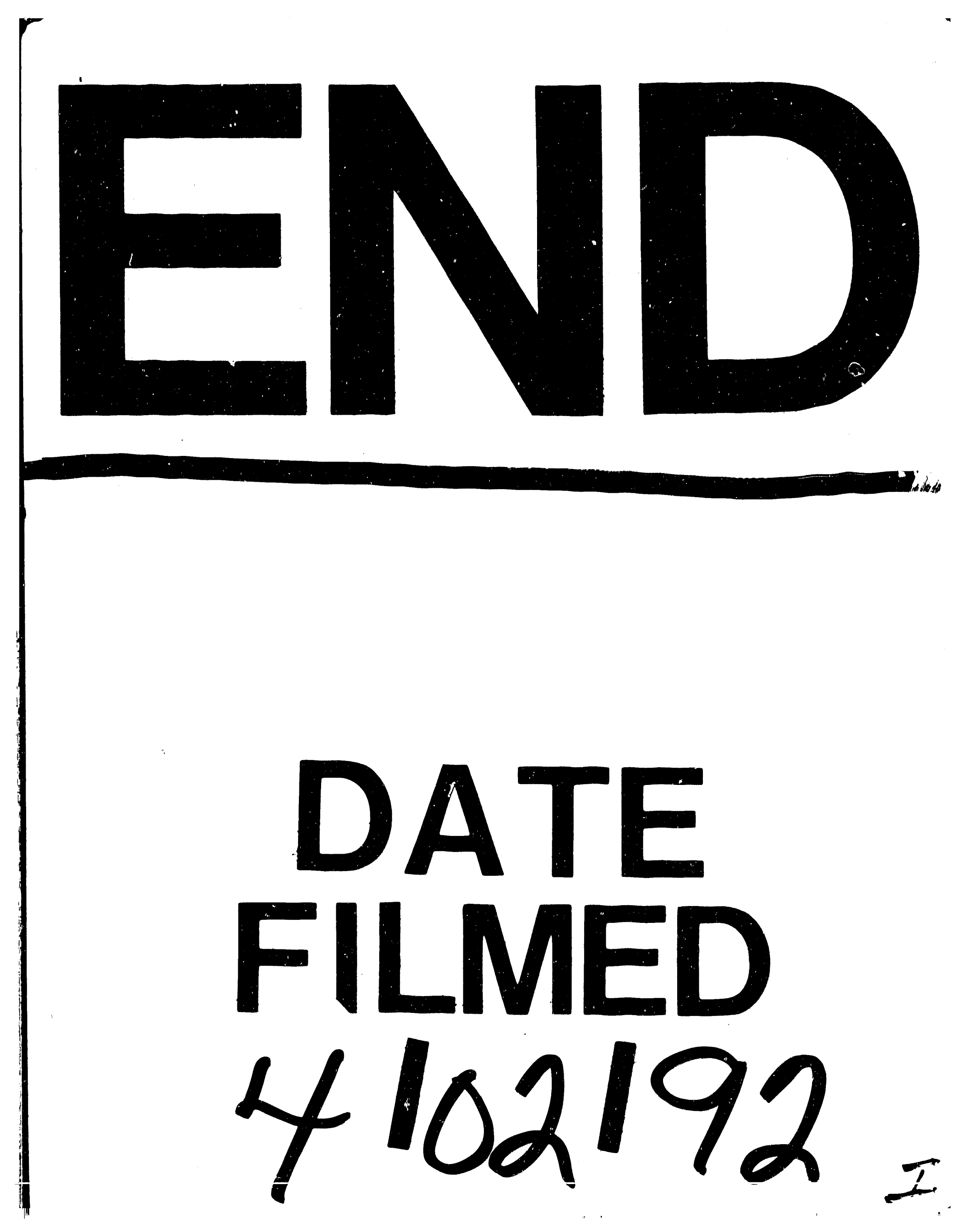


